\title{
Retinal locus for scanning text
}

\author{
George T. Timberlake, PhD; ${ }^{1-2 *}$ Manoj K. Sharma, MD; ${ }^{1}$ Susan A. Grose, BS; ${ }^{1}$ Joseph H. Maino, OD, FAAO $^{1-2}$ \\ ${ }^{1}$ Kansas City Veterans Affairs Medical Center, Kansas City, MO; ${ }^{2}$ Department of Ophthalmology, University of Kansas \\ Medical Center, Kansas City, KS
}

\begin{abstract}
A method of mapping the retinal location of text during reading is described in which text position is plotted cumulatively on scanning laser ophthalmoscope retinal images. Retinal locations that contain text most often are the brightest in the cumulative plot, and locations that contain text least often are the darkest. In this way, the retinal area that most often contains text is determined. Text maps were plotted for eight control subjects without vision loss and eight subjects with central scotomas from macular degeneration. Control subjects' text maps showed that the fovea contained text most often. Text maps of five of the subjects with scotomas showed that they used the same peripheral retinal area to scan text and fixate. Text maps of the other three subjects with scotomas showed that they used separate areas to scan text and fixate. Retinal text maps may help evaluate rehabilitative strategies for training individuals with central scotomas to use a particular retinal area to scan text.
\end{abstract}

Key words: eccentric viewing, fixation, fixation PRL, preferred retinal locus, reading, reading rehabilitation, retinal function mapping, scanning laser ophthalmoscope, text scanning, text-scanning PRL

\section{INTRODUCTION}

When individuals without vision loss read, their fast saccadic eye movements sequentially shift the characters in a line of text onto the fovea, the retinal area of highest acuity, where they are held in place and visually processed. This typical reading process is disrupted when foveal vision is lost because of macular disease. Normal reading rates usually exceeding 150 words a minute can drop to approximately 25 words a minute or less [1]. Individuals with foveal vision loss resulting in dense central scotomas use a peripheral retinal area (preferred retinal locus [PRL]) adjacent to the scotoma to fixate and inspect small visual objects, such as single letters. They may also use this fixation PRL (fPRL) to scan extended sentences and paragraph text. Over the past 25 years, various rehabilitative strategies have been proposed for enhancing use of eccentric (i.e., PRL) viewing and improving reading rates [2-4]. These rehabilitative measures have met with some success but may be less than optimal. One impediment to the development of effective reading rehabilitation strategies has been the lack of a means to adequately characterize the retinal area used for scanning text.

Determining the fPRL for inspecting small visual objects such as fixation targets, letters, or short words is relatively straightforward because these visual stimuli are spatially discrete. Consequently, their positions on the retina are readily characterized with multiple scanning laser ophthalmoscope (SLO) images. Mapping the retinal

\footnotetext{
Abbreviations: $\mathrm{fPRL}=$ fixation preferred retinal locus, $\mathrm{fps}=$ frames per second, $\mathrm{PRL}=$ preferred retinal locus, $\mathrm{SD}=$ standard deviation, SLO = scanning laser ophthalmoscope, $\mathrm{tPRL}=$ text-scanning PRL.

* Address all correspondence to George T. Timberlake, PhD; Department of Ophthalmology, University of Kansas Medical Center, 3901 Rainbow Boulevard, Kansas City, KS 66160-7379; 913-588-6647; fax: 913-381-7726.

Email: gtimberl@kumc.edu

DOI: 10.1682/JRRD.2005.06.0102
} 
locus for scanning multiple words in paragraphs is more problematic, however, since the text is spatially extensive and occupies a much larger retinal area. For example, Figure 1 shows an SLO image of paragraph text on a retina with a hypothetical scotomatous central lesion. The text extends beyond the scotoma on all sides. In this hypothetical example, the retinal area being used to inspect the text at the moment the SLO image was made is impossible to determine. As shown by the ovals with question marks, many possibilities exist (Figure 1). One cannot assume that the fPRL for inspecting small objects was also used for scanning the extended text.

Here we report a method for mapping the retinal area that subjects used to scan text during reading. This method is based on an earlier graphical method [5]. The present method cumulatively plots text position on an SLO retinal image. In the cumulative plot, retinal areas that contained text most often will be the brightest, and those that contained text least often will be the darkest. In this way, we can determine the retinal region that most often contains text. In essence, the method is like a time exposure of film in a camera. Suppose that a camera, like the eye, jumps (saccades) from one point to another on an illuminated image in an otherwise dark environment, for example, an illuminated advertising sign at night. The film in the camera (the "retina") will be most exposed in the center because it is most often pointed at part of the illuminated image. Other peripheral parts of the film will

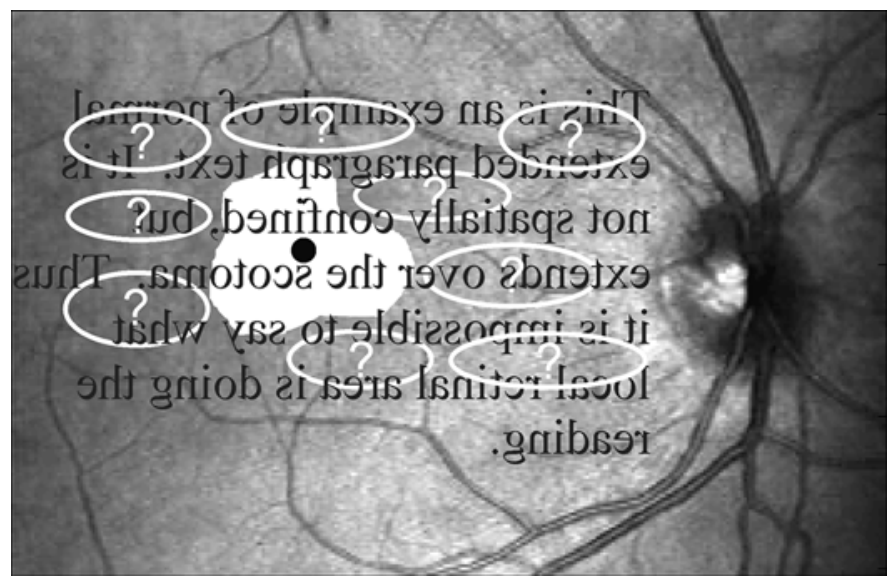

Figure 1.

Hypothetical scanning laser ophthalmoscope image of text on retina. Image illustrates problem of determining which retinal area scans spatially extensive text. Black dot is position of nonfunctional foveal center. Irregular white area is scotomatous area. Ovals with question marks are possible retinal areas used for reading text. be less exposed. When the film is developed, one will find a bright illuminated central portion surrounded by increasingly dimmer regions.

\section{METHODS}

\section{Subjects}

Participants were eight control subjects without vision loss and eight subjects who had had macular degeneration and central vision loss with scotomas in both eyes for more than 1 year. All subjects provided written informed consent, and the study was approved by the Kansas City Department of Veterans Affairs Medical Center Human Subjects Committee.

\section{Retinal Mapping}

\section{Stimuli and Procedures}

Control subjects viewed stimuli in a Rodenstock SLO (Fourward Technologies, Inc; Buena Vista, Virginia) using their dominant eye, which was determined by the "hole in card" test; the other eye was patched. Subjects with scotomas used their preferred eye for reading; the other eye was patched. Subjects stabilized their heads on a chin/forehead rest that we designed and attached to the SLO. To measure the retinal location of fixation, we presented a cross or square in the center of the SLO laser-beam raster. For control subjects, the cross or square was approximately $0.2^{\circ} \times 0.2^{\circ}$. For the subjects with scotomas, the size of the fixation stimulus varied from $0.5^{\circ} \times 0.5^{\circ}$ to $2.0^{\circ} \times 2.0^{\circ}$. Fixation stimuli were the same brightness as the text stimuli. Subjects were asked to "look at the square (or cross) as carefully as possible" while the experimenter counted for approximately $12 \mathrm{~s}$. We recorded two or three such fixation trials for each subject.

To measure the retinal location of text, we presented three-line sentences, written at an elementary school level in Times New Roman font, in the SLO helium-neon laser-beam raster. The text was centered in the raster and presented when the subjects signaled that they were prepared to read. Subjects were instructed to fixate until the text appeared, read the text silently, and then read the sentence aloud. Character size was $\sim 0.7^{\circ} \times \sim 0.7^{\circ}$ for all control subjects. Before the reading trials began, we determined the text font size for subjects with scotomas by increasing the character size in 0.1 log steps until each 
subject was able to correctly read the sentence. Each subject read three different sentences. SLO images were videotaped (30 frames per second [fps]) throughout each session.

\section{Data Analysis}

For text-scanning measurement, we digitized the videotaped SLO images of subjects reading one of the three sentences (the one with the least image loss) at a rate of $30 \mathrm{fps}$ with half-size format $(305 \times 188$ pixels) for convenient storage and processing. Figure 2 is an example of every twentieth digitized frame (i.e., every $0.67 \mathrm{~s}$ ) of a control subject reading the sentence, "She wanted to show us the new toys she got for her birthday.” (All SLO images in this article are oriented as a projection of the retina onto the visual field. Thus, the superior retina is on the bottom of the image and the nasal retina is to the side of the optic disk.) A blood vessel landmark or other small retinal feature (Figure 2, black circles) was measured on each digitized SLO image using Matrox Inspector software (Matrox Electronic Systems, Ltd; Dorval, Québec, Canada). Measurements were made on a single sentence for each subject. The $x, y$ (horizontal, vertical) pixel coor-
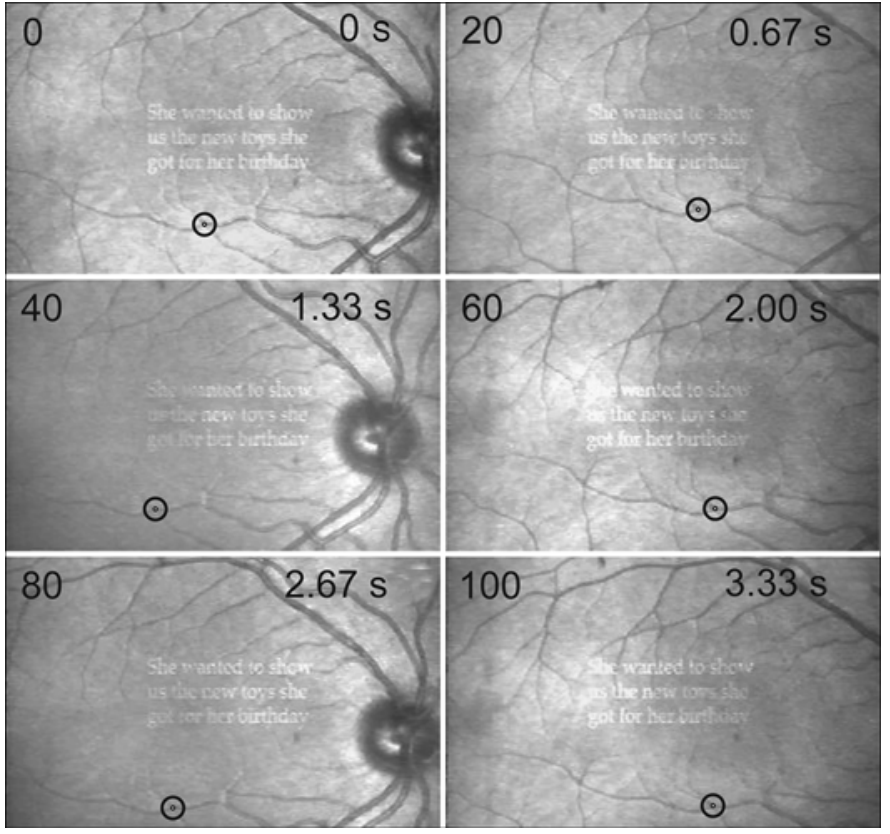

Figure 2.

Sequence of digitized scanning laser ophthalmoscope images of subject without vision loss reading three-line sentence. Every twentieth digitized frame shown. Frame number shown in upper-left corner, time in upper-right corner. Black circles show measured retinal vessel landmark. Superior retina at bottom of each image. dinates of the landmark were stored for later analysis. All $103 x, y$ coordinates for the subject in Figure 2 are plotted as a function of time in Figure 3. The plot shows "staircase" reading eye movements with fixations of $\sim 300 \mathrm{~ms}$ in the horizontal direction and saccades that moved the fovea along the line of text. The subject executed two saccades to return to the beginning of the second and third lines of text (Figure 3). Two of the eight control subjects exhibited such multiple return saccades, while the other six made a single return saccade.

For fixation measurement, we digitized one videotaped fixation trial as described for text scanning. The $x, y$ coordinates of a retinal vessel landmark were measured every third video frame (i.e., every $0.1 \mathrm{~s}$ ) throughout the fixation trial. This resulted in 100 to $114 x, y$ coordinates for each subject.

\section{Text Mapping}

Maps that showed retinal text location while subjects read a sentence were created in several steps. First, we digitized an image of the text in the SLO raster and turned individual text characters into solid white (gray

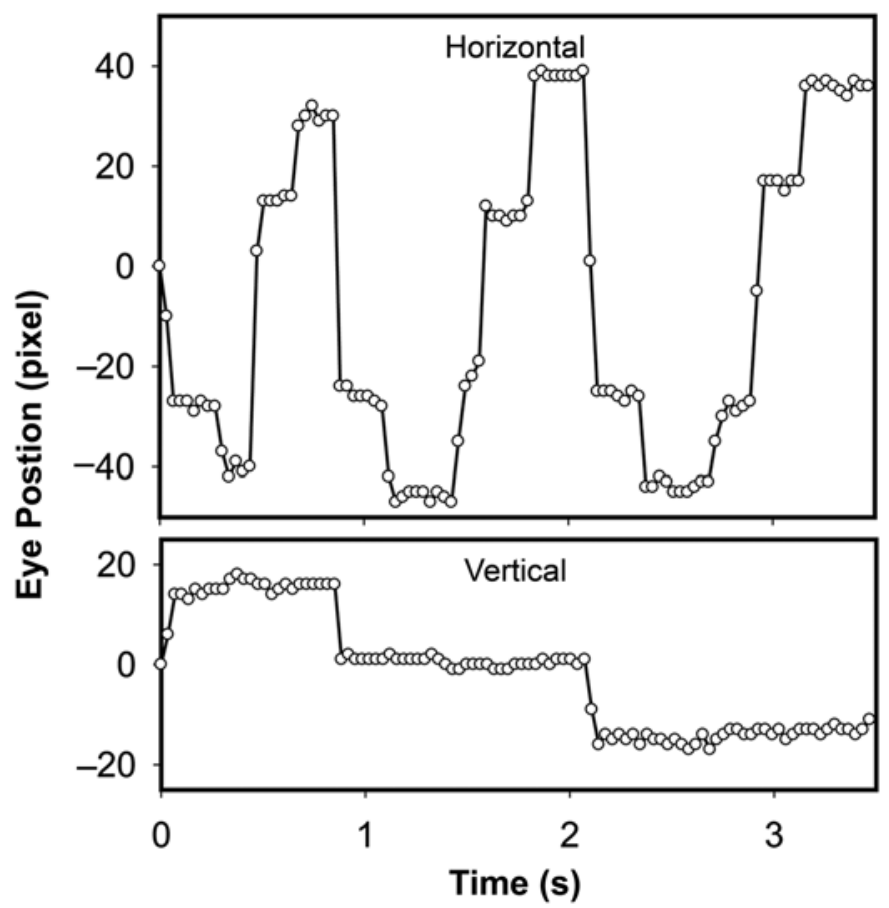

Figure 3.

Measured $x$ (horizontal) and $y$ (vertical) positions of retinal vessel landmark of subject without vision loss during reading sequence. Each open circle represents one $x$ or $y$ measurement. 
scale level 255) rectangles on a black (gray scale level 0) background using Photoshop (Adobe Systems Inc, San Jose, California) (Figure 4(a)). Letters were changed to solid rectangles because the shape of individual letters is irrelevant and the blocked image ultimately makes a more visible and quantifiable text map. Second, we wrote a program ("ShiftAdd") in MATLAB (The MathWorks, Inc, Natick, Massachusetts) that shifted the binary text image in the $x, y$ directions based on the previously recorded $x, y$ landmark coordinates (e.g., Figure 3). The $x, y$ coordinates shift the binary text image $x$ pixels in the horizontal direction and $y$ pixels in the vertical direction. After each binary image shift, the intensity levels of pixels at each $x, y$ coordinate in the original and shifted images are added. Thus, the new image becomes brighter where the text
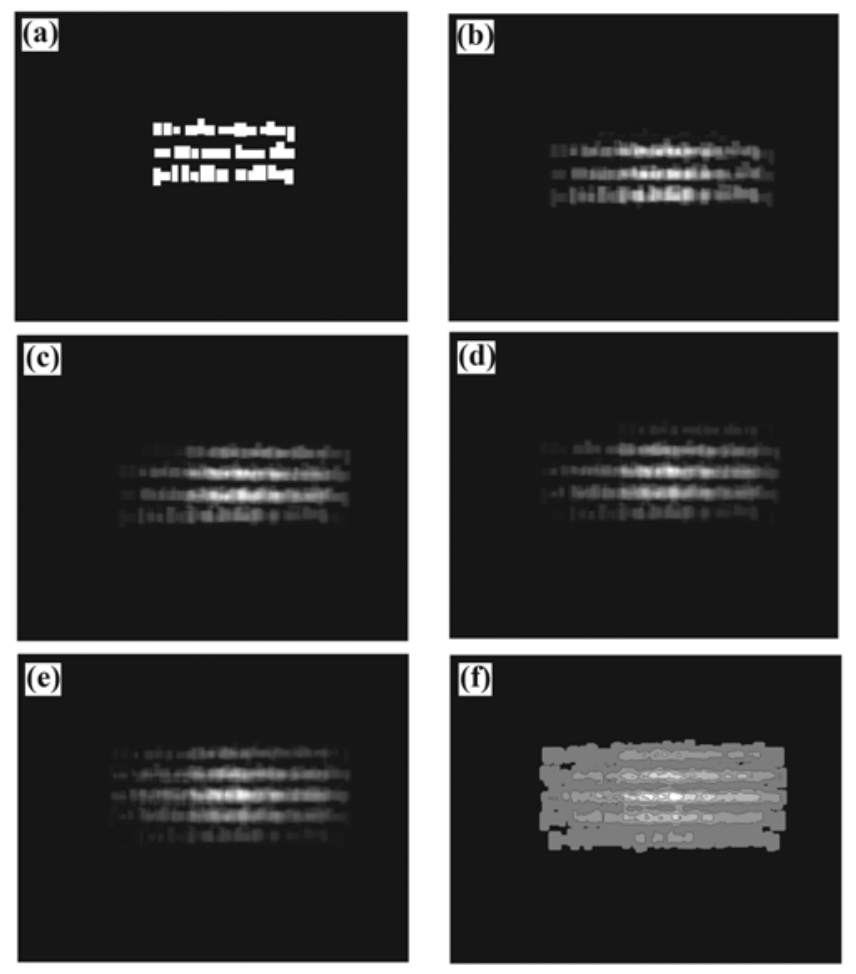

Figure 4.

Method of producing text maps. (a) Text image with characters replaced by solid white rectangles to form binary text image. Computer program shifted binary text image $x$ pixels horizontally and $y$ pixels vertically based on recorded retinal landmark coordinates. After each shift, pixel intensity at each $x, y$ coordinate of original and shifted images is added. Remaining panels show text image after (b) 25 shifts and adds, (c) 50 shifts and adds, (d) 75 shifts and adds, and (e) all 103 shifts. (f) Contour map of image shows quintiles of intensity. blocks overlapped but remains the same where the text blocks did not overlap. With each successive shift and addition of the text-block stimulus, some areas become brighter. Figure 4 shows the images that resulted from 25 (Figure 4(b)), 50 (Figure 4(c)), and 75 (Figure 4(d)) "shifts" and "adds." Figure 4(e) shows the final image after all 103 shifts. In these figures, image brightness (i.e., gray scale level) at any point is directly proportional to the time that text characters were on that point. The brightness of the final image can be represented as a contour plot (Figure 4(f)). The contour plot shows the proportion in quintiles of the total reading time that text characters occupied a retinal area. Thus, the largest, darkest gray area shows the retinal region occupied by text 1 to 20 percent of the time; the next lightest gray area shows where text occupancy was 21 to 40 percent. The brightest, white area shows the region that was occupied by text 81 to 100 percent of the time. The black area represents no text occupancy.

\section{Master Retinal Text Image}

We used the same method for creating text maps to create a retinal image on which to plot the text map. Beginning with the first SLO image in the reading sequence, the computer program shifted each subsequent image by the $x, y$ coordinates and added it to the previous image. Figure 5(a) shows the result of shifting and adding all 103 SLO images of the reading sequence shown partially in Figure 2. The retinal image is clear, which indicates that the frames were accurately shifted before adding. Figure 5(b) shows the image that results when the frames are not shifted before adding. Only the text, which is stationary in each frame, remains clearly visible.

The text map in Figure 4(f) corresponds exactly to the retinal position in the retinal image in Figure 5(a). This correspondence occurs because the computer programs

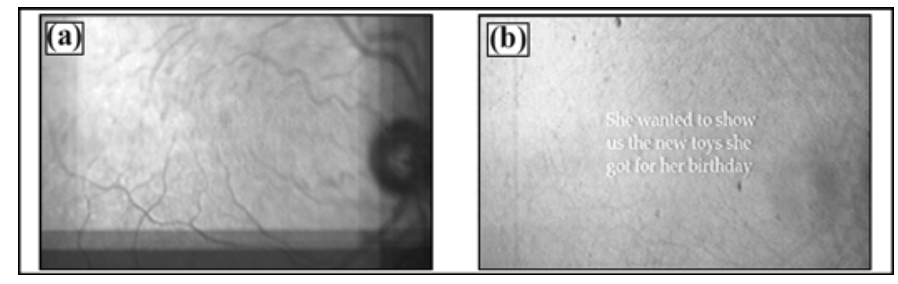

Figure 5.

(a) Image of retina after shifting and adding 103 individual scanning laser ophthalmoscope images during reading sequence in Figure 2. (b) Effects of not shifting images prior to addition. 
sequentially shift each SLO image so that it lines up with the first SLO video frame and sequentially shifts the binary text block image the same amount. Since the text map image (Figure 4(f)) corresponds exactly to the final shifted retinal image (Figure 5(a)), the two can be superimposed, showing the retinal location of text scanning (Figure 6).

\section{Fixation Mapping}

We used the same method for creating a map of retinal text position to make a retinal map of the location of a fixation stimulus while the subject fixated. Figure 7 illustrates the method with the same subject from Figures 2-6. First, we digitized a binary image of the fixation stimulus in the SLO raster (Figure 7(a)). Second, the computer program shifted and added the binary image using the $x, y$ coordinates that were measured from SLO images during $10 \mathrm{~s}$ fixation (Figure 7(b)). Just as in the text map, the image became brighter where the shifted fixation stimulus overlapped on successive frames; thus, the brightness (i.e., gray scale level) at any point is directly proportional to the time that the fixation stimulus was on that point (Figure 7(c)). The intensity map is presented as a contour map in Figure 7(d). Only one contour enclosed all the retinal areas that contained the fixation stimulus.

We used the same method for producing a master image of the retina for the text map for producing a retinal image for the fixation map. Beginning with the first digitized SLO image of the fixation sequence, the computer program shifted each subsequent image using the

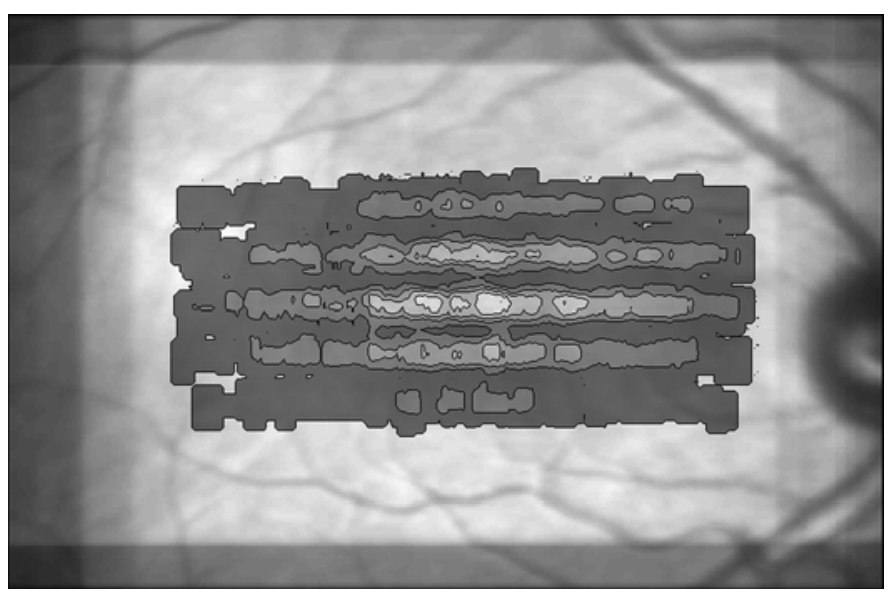

Figure 6.

Retinal location of text scanning. Text map in Figure 4(f) superimposed on corresponding retinal position of shifted retinal image in Figure 5(a). fixation $x, y$ coordinates and added it to the previous image. Figure 8(a) shows the result of shifting and adding the 102 digitized SLO images during fixation. The fixation map in Figure 7(d) corresponds precisely to the retinal image in Figure 7(a) and thus can be overlaid, showing the retinal location of fixation for this subject (Figure 8(b)).

\section{Complete Retinal Map}

We added the retinal fixation area in Figure 8 to the corresponding retinal text map by overlaying the two images, aligning the retinal vasculature, and transferring
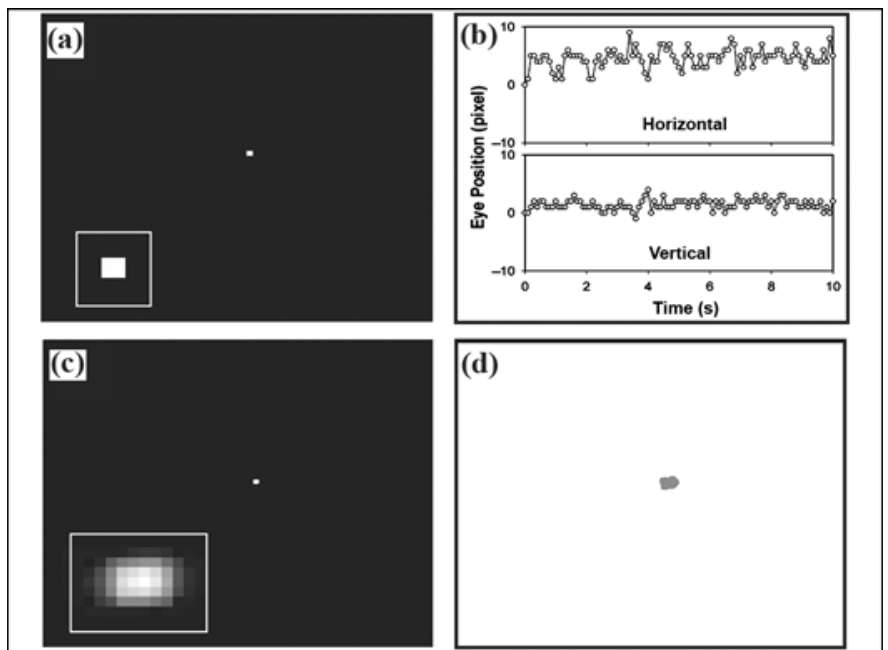

Figure 7.

Method of retinal fixation mapping. (a) Binary image of fixation stimulus. Inset shows magnified fixation stimulus. (b) Horizontal and vertical positions of retinal vessel landmark during $10 \mathrm{~s}$ fixation. (c) Result of successively shifting original binary image according to landmark coordinates and adding images. Inset shows magnified fixation map. (d) Contour map of resulting image that shows all retinal positions of fixation stimulus.

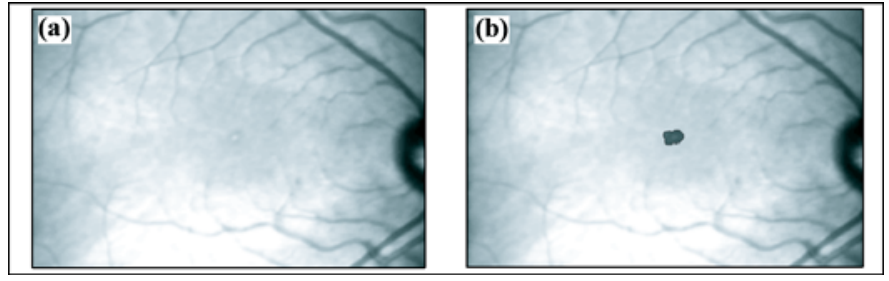

\section{Figure 8.}

(a) Master retinal image for plotting fixation position produced by shifting and adding 102 digitized scanning laser ophthalmoscope images. (b) Retinal location of fixation contour map. 
the position of the fixation area onto the text map. This process is illustrated in Figure 9 with images from the same subject from Figures 2-6. Before alignment, we superimposed the retinal image showing the fixation area on the retinal text map in an overlay plane using Photoshop (Figure 9(a)). The fixation image was then moved with the computer mouse until the retinal vasculature of the two images was aligned and the retinal fixation area was traced onto the text map (Figure 9(b)).

The retinal area for scanning text and fixation can be presented in a "cartoon" format in which the retinal vasculature and optic disk are traced from the retinal image onto an overlay plane (Figure 10). In addition, the gray scale contour map of text occupancy can be changed to a color contour map for better visualization.

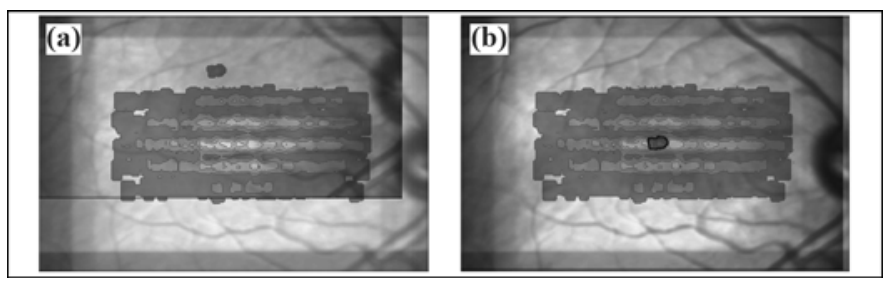

Figure 9.

Procedure for creating retinal map that shows location of fixation area and retinal area for scanning text. (a) Offset image of retina with fixation location in overlay plane superimposed on retinal image with text map. (b) Fixation and text map images aligned and fixation area traced onto text map (black outline).

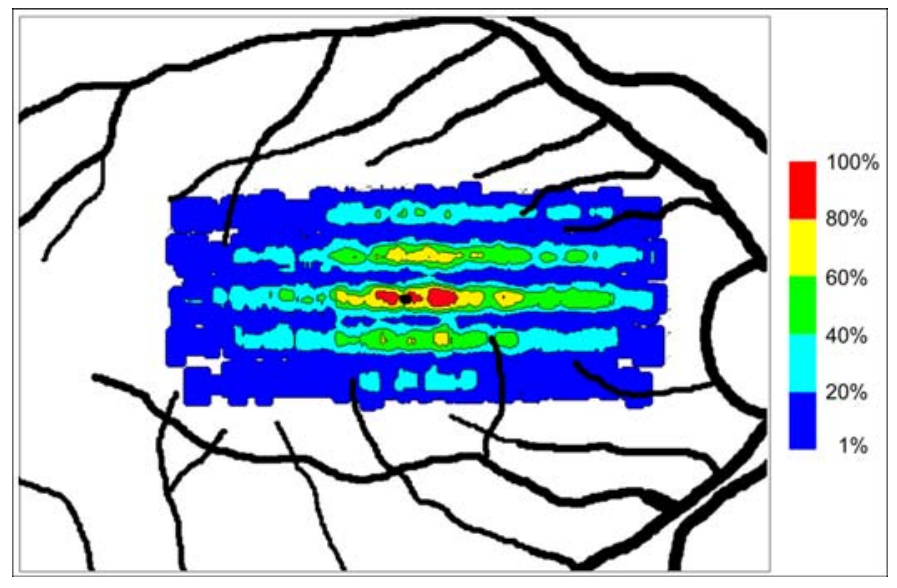

Figure 10.

Example of color cartoon map of image in Figure 9(b). Same contours but in color. Black area in center is foveal fixation position. Color scale on right shows percentage of time text occupied each retinal region.

\section{Foveal Location}

Identifying foveal location in SLO images of retinas with macular degeneration is usually impossible [6]. Consequently, we estimated foveal location by measuring where subjects without vision loss placed the fixation stimulus relative to their optic disks. Specifically, we measured the distances (in pixels) from the inferior and temporal disk margins to the fixation stimulus in SLO images of 25 subjects without vision loss who had participated in previous studies. We calculated the mean and standard deviation (SD) of the $x, y$ fixation locations relative to the optic disk for all 25 subjects. The mean foveal fixation position could then be plotted relative to the optic disk of subjects whose foveas were not visible. A $\pm 2 \mathrm{SD}$ box was drawn around the mean position (Figure 11).

\section{RESULTS}

\section{Control Subjects}

Text and foveal fixation maps of the eight control subjects are shown in Figure 12. The most salient features of these maps are (1) they all have a five-horizontalline structure, (2) the area of highest text occupancy $(81 \%-100 \%)$ is in the center of the map, and (3) the foveal fixation position is in the center of the region of highest text occupancy. First, the five-line structure occurs because when reading begins, the fovea is on the top line of text, hence two lines are "below" the fovea in

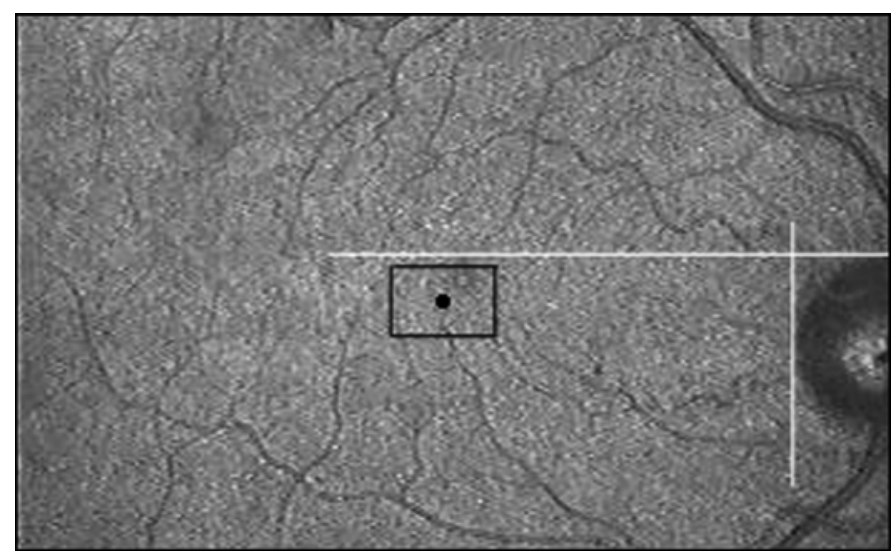

Figure 11.

Example of estimated foveal position. White lines show inferior and temporal edges of optic disk. Black dot is mean $x, y$ (horizontal, vertical) position of foveal fixation area relative to optic disk edges. Black box is \pm 2 standard deviations in $x$ and $y$ directions. 


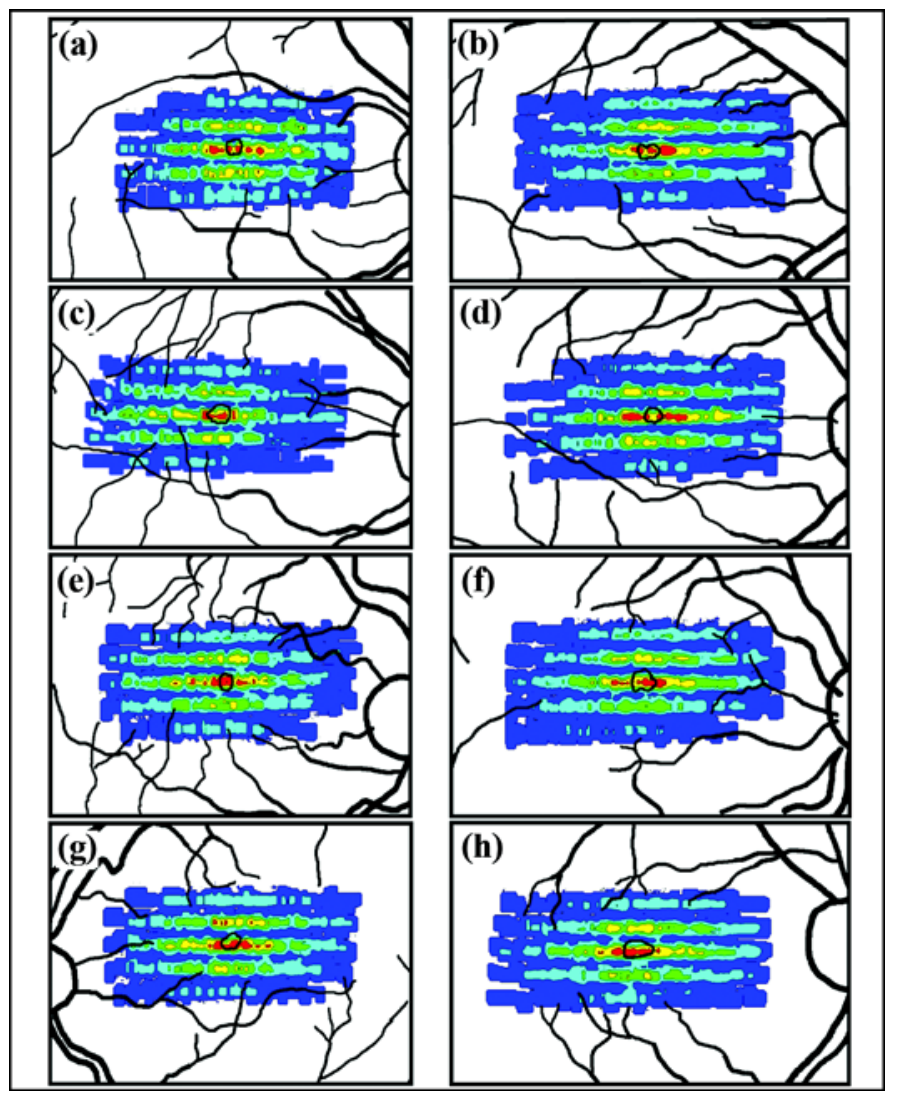

Figure 12.

Retinal text maps for eight subjects without vision loss. Black line outlines foveal fixation position. Contours represent quintiles of text occupancy. See Figure 10 for color scale.

the superior retina. When reading ends, the fovea is on the bottom line of text; hence, two lines are "above" the fovea in the inferior retina. Thus, altogether five lines appear in the text plot. Second, the retinal area with the top quintile (81\%-100\%) of text occupancy (Figure 12, red areas) is confined to a relatively small central area of the center line of the text map. The red area thus shows the retinal region that most frequently contained text characters. Third, the foveal fixation position is in the center of the map and the contour area of highest text occupancy. Thus, text was most often placed on the fovea and to the left and right of the foveal center. This text placement is not surprising since the fovea is generally accepted to scan text during normal reading. The maps show this in a direct graphical manner.

\section{Subjects with Scotomas}

Retinal text maps of the eight subjects with bilateral, dense macular scotomas fell into three categories: (1) the
fPRL was near the estimated foveal location and was used to scan text, (2) the fPRL was distant from the estimated foveal location and was used to scan text, and (3) the fPRL was distant from the fovea but was not used to scan text; another retinal area scanned text. Each of these categories is subsequently described.

\section{fPRL Near Fovea and Used to Scan Text}

Three of the subjects with scotomas had fPRLs that were adjacent to or partially overlapping the estimated foveal location; they used the fPRL to scan text (Figure 13). Thus, these subjects most likely fixated and scanned text with a perifoveal retinal area. One subject's fPRL (Figure 13(d)) was approximately the same area as the control subjects', while the other two subjects exhibited a much larger fPRL (Figure 13(b) and (f)). The retinal text maps all exhibited the five-line structure (Figure 13(a), (c), and (e), arrows) seen in the control subjects' text maps; additionally, the fPRL overlapped the retinal area of highest text occupancy. Thus, these three subjects apparently scanned the text with the fPRL. One subject (Figure 13(a)) exhibited a retinal text map very similar to that of the control subjects. This text map was approximately twice
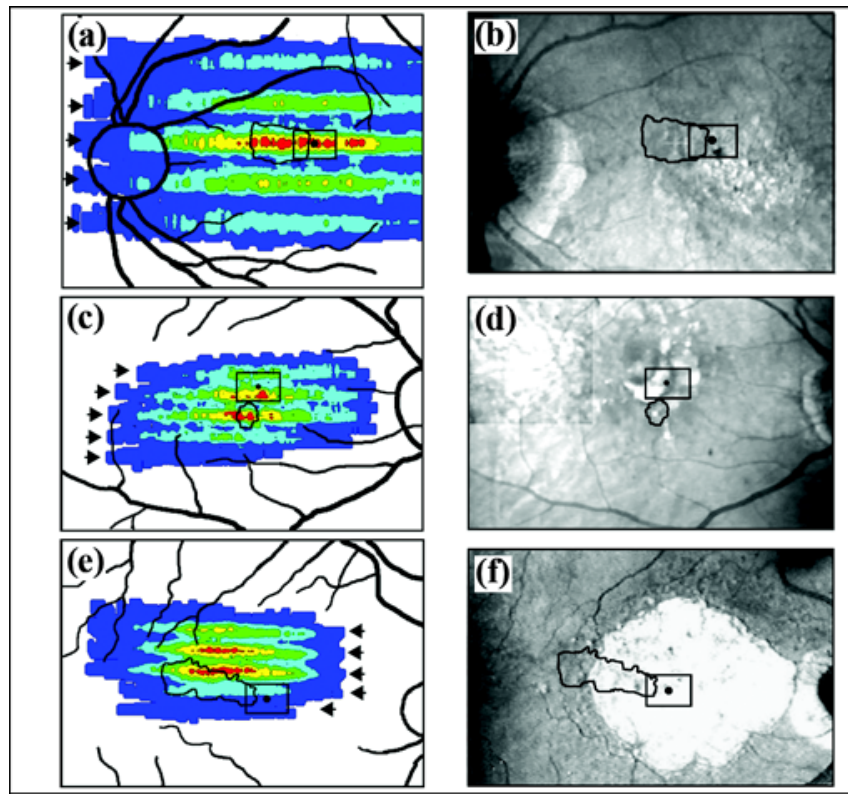

Figure 13.

(a), (c), and (e) Retinal text maps and (b), (d), and (f) fixation maps of three subjects with scotomas whose fixation preferred retinal loci (irregular black outlines) were contiguous with estimated foveal area (rectangular black outlines) and were used to scan text. Arrows in (a), (c), and (e) designate horizontal line structure. See Figure 10 for color scale. 
as large as the control subjects' text maps because larger text characters were used. This text map shows that the area of highest text occupancy was confined to the center of the central line. In the other two subjects' text maps, however, two of the lines had areas of highest text occupancy (Figure 13(c) and (e), red areas). This type of text map occurs when the retinal locus used for scanning text spends more time on one or two lines than on a third; it does not indicate two text-scanning PRLs (tPRLs) (to be described in "Simulations" section).

\section{fPRL Distant from Fovea and Used to Scan Text}

Two subjects with scotomas exhibited fPRLs that were relatively distant from the estimated foveal area (Figure 14(b) and (d)). In each case, the fPRL also overlapped the retinal area of highest text occupancy (Figure 14(a) and (c)). Neither subject had a normal five-line text map. The first subject exhibited a relatively compact text map, but only four lines are apparent and two of the lines contained text in the top quintile of occupancy (Figure 14(a) and (b)). This four-line text map occurred because the SLO image was degraded when the subject scanned the first line of text, and consequently, no retinal landmark measurements were made (to be described in "Simulations" section). The second subject had a three-line text map, and the fPRL and retinal area of highest text occupancy did not completely overlap (Figure 14(c) and (d)). Thus, the second subject may have a separate fPRL and tPRL.

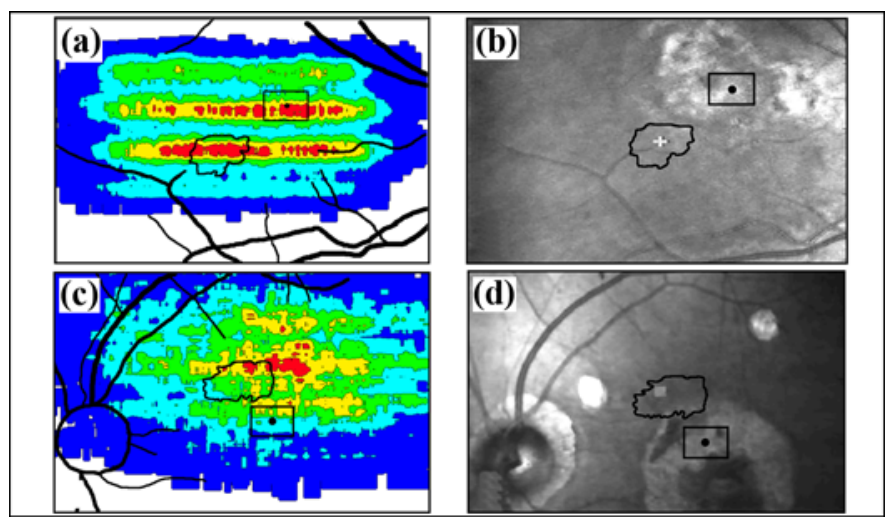

Figure 14.

(a) and (c) Retinal text maps and (b) and (d) fixation maps of two subjects with scotomas whose fixation preferred retinal loci (irregular black outlines) were clearly separate from estimated foveal position (rectangular black outlines). See Figure 10 for color scale.

\section{fPRL Distant from Fovea but Not Used to Scan Text}

Three of the subjects with scotomas scanned text with a different retinal area than they used to fixate. That is, they did not use their fPRL for scanning text (Figure 15) but, rather, used a distinct tPRL. One of these subjects exhibited a very clear five-line text map in which the top quintile of text occupancy was positioned vertically between the estimated foveal area and the fPRL (Figure 15(a) and (b)). The second subject had an fPRL quite distant superiorly from the estimated foveal position (Figure 15(c) and (d)). The area of highest text occupancy lay between the estimated foveal area and the fPRL. The text map is somewhat fragmented, but five lines can be delineated. This type of text map can arise from increased random variability in PRL placement on the text. The third subject who scanned text with a separate tPRL is shown in Figure 15(e) and (f). The area of highest text occupancy of this five-line map was positioned vertically between the estimated foveal area and the fPRL.

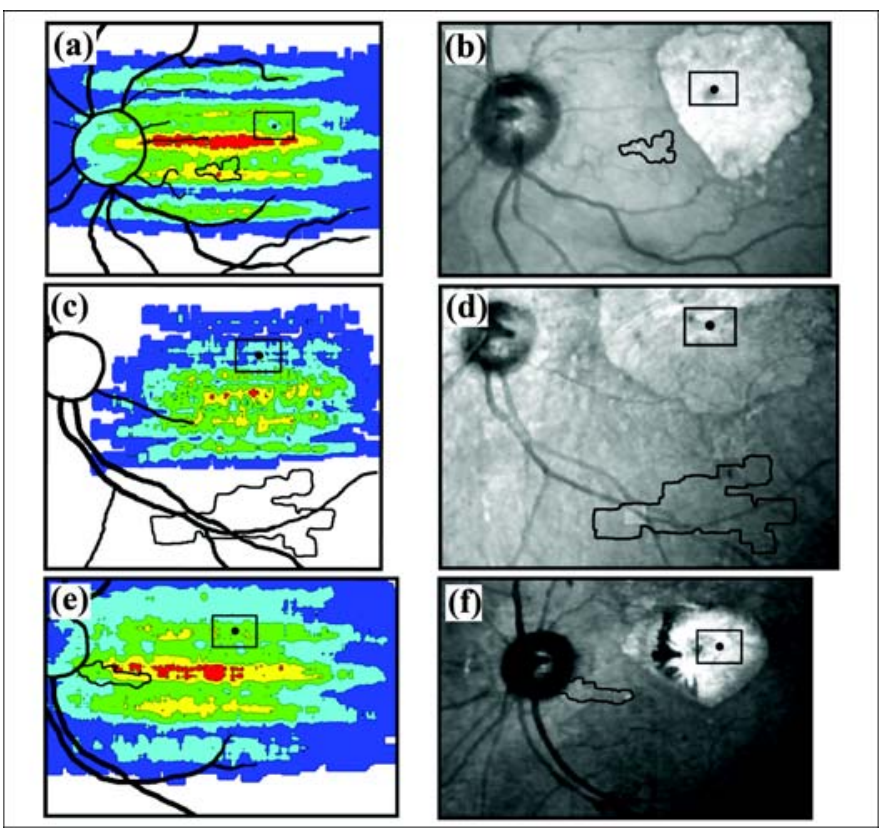

\section{Figure 15.}

(a), (c), and (e) Retinal text maps and (b), (d), and (f) fixation maps of three subjects with scotomas who scanned text using a retinal area different from their fixation preferred retinal locus (irregular black outlines). Rectangular black outlines ((b), (d), and (f)) show estimated foveal position. See Figure 10 for color scale. 


\section{Simulations}

We developed computer simulations in MATLAB to investigate text map formation. The first simulation constrained the tPRL so that it landed only on text characters in the three-line sentence. The exact landing spot of the tPRL on a letter could be randomly varied within the letter area. The character on which the tPRL landed was chosen randomly; thus, the sequence of "saccades" from one character to another was random. In addition, the percentage of time that the tPRL spent on each of the three text lines could be varied. Text maps produced by the first simulation are shown in Figure 16. Each text map was produced by 150 shifts and adds as described earlier (i.e., the tPRL shifted onto text characters 150 times). The typical text map in Figure 16(a) resulted when the tPRL spent an equal percentage of time on each line (i.e., 33.3\% on each line). The five-line structure of this text map is almost identical to that seen for the control subjects (Figure 12) and two of the subjects with scotomas (Figures 13(a) and 15(a)). When the tPRL spent an unequal percentage of time on each line, text maps similar to those of some of the subjects with scotomas resulted. For example, when the percentages of time on the first, second, and third lines were 0,34 , and 66 percent, respectively (i.e., the tPRL never landed on the first line), the four-line text map in Figure 16(b) resulted. This text map is quite similar to that of the scotoma subject's text map in Figure 14(a). A review of the videotape for this subject revealed that the SLO image was degraded at the start of the reading sequence. Consequently, landmark measurements were not made while the subject was scanning the first line of text, resulting in the four-line text map.

By adjusting the percentage of time spent on each text line, we could simulate text maps of other scotoma subjects. Thus, when the percentages on each line were 10 , 40, and 50 percent, respectively, a five-line text map (Figure 16(c)) was generated that was similar to that of the scotoma subject in Figure 13(c). Finally, when the percentages of time on each line were 35,55 , and 10 percent, respectively, the five-line text map (Figure 16(d)) was similar to that of the scotoma subject in Figure 13(e).

If random retinal shifts unrelated to text characters were to produce realistic text maps, then the retinal textmapping technique would be questionable. A second computer simulation addressed this possibility. In the first simulation, the order of fovea and/or PRL shifts was random but the positions were constrained to text charac-
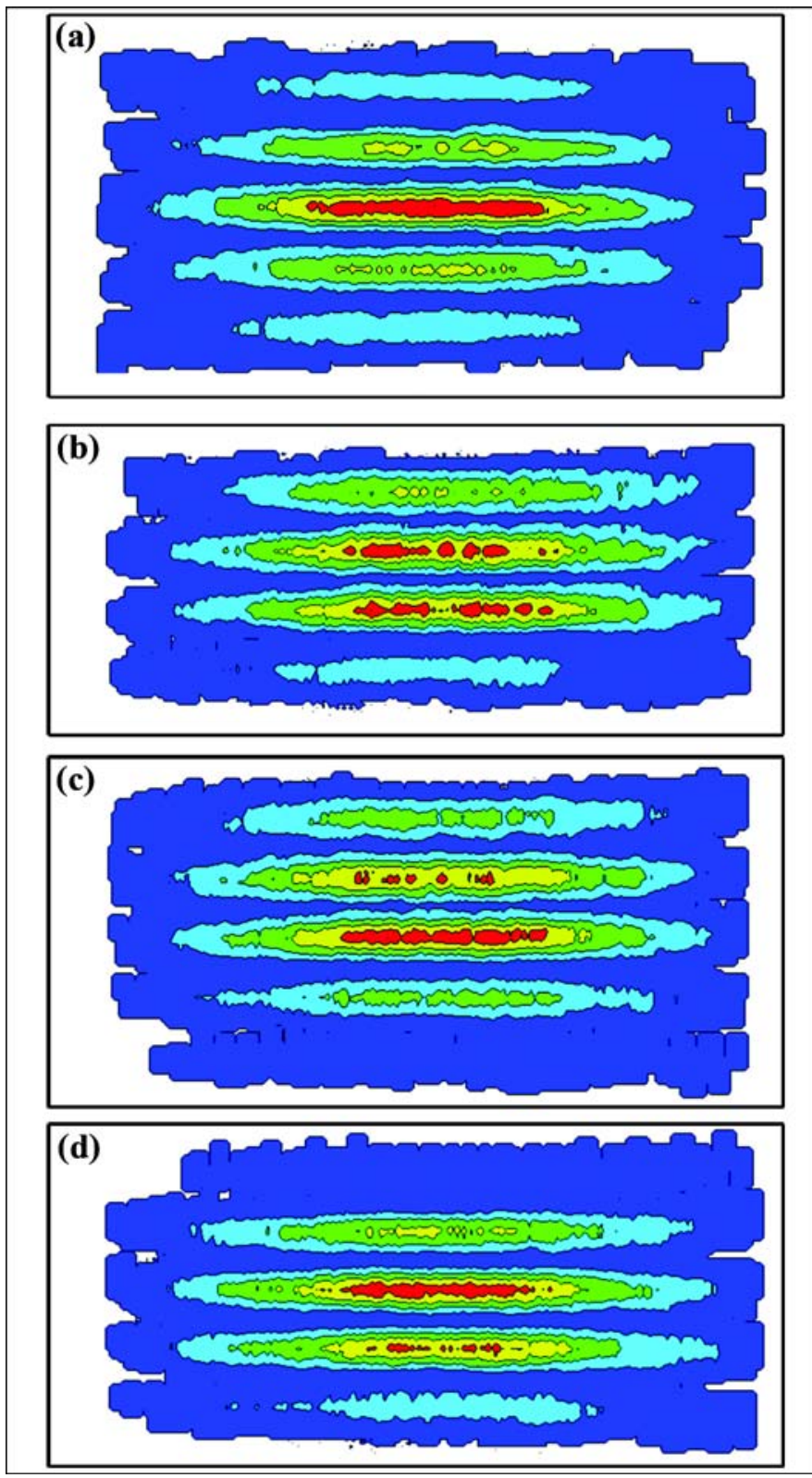

Figure 16.

Text maps produced by computer simulations that varied percentage of time spent by fovea or preferred retinal locus on first, second, and third lines of text: (a) $33.3 \%$ on each line; (b) $0 \%$, 34\%, and $66 \%$; (c) 10\%, 40\%, and 50\%; and (d) 35\%, 50\%, and 10\%. See Figure 10 for color scale.

ters. In this second simulation, both the order and positions of fovea and/or PRL shifts were random but constrained to a certain rectangular area around the text. Examples of "random scan" maps are shown in Figure 17. For the map in Figure 17(b), 150 shifts were performed in random order and position but constrained to a $112 \times 48$ 

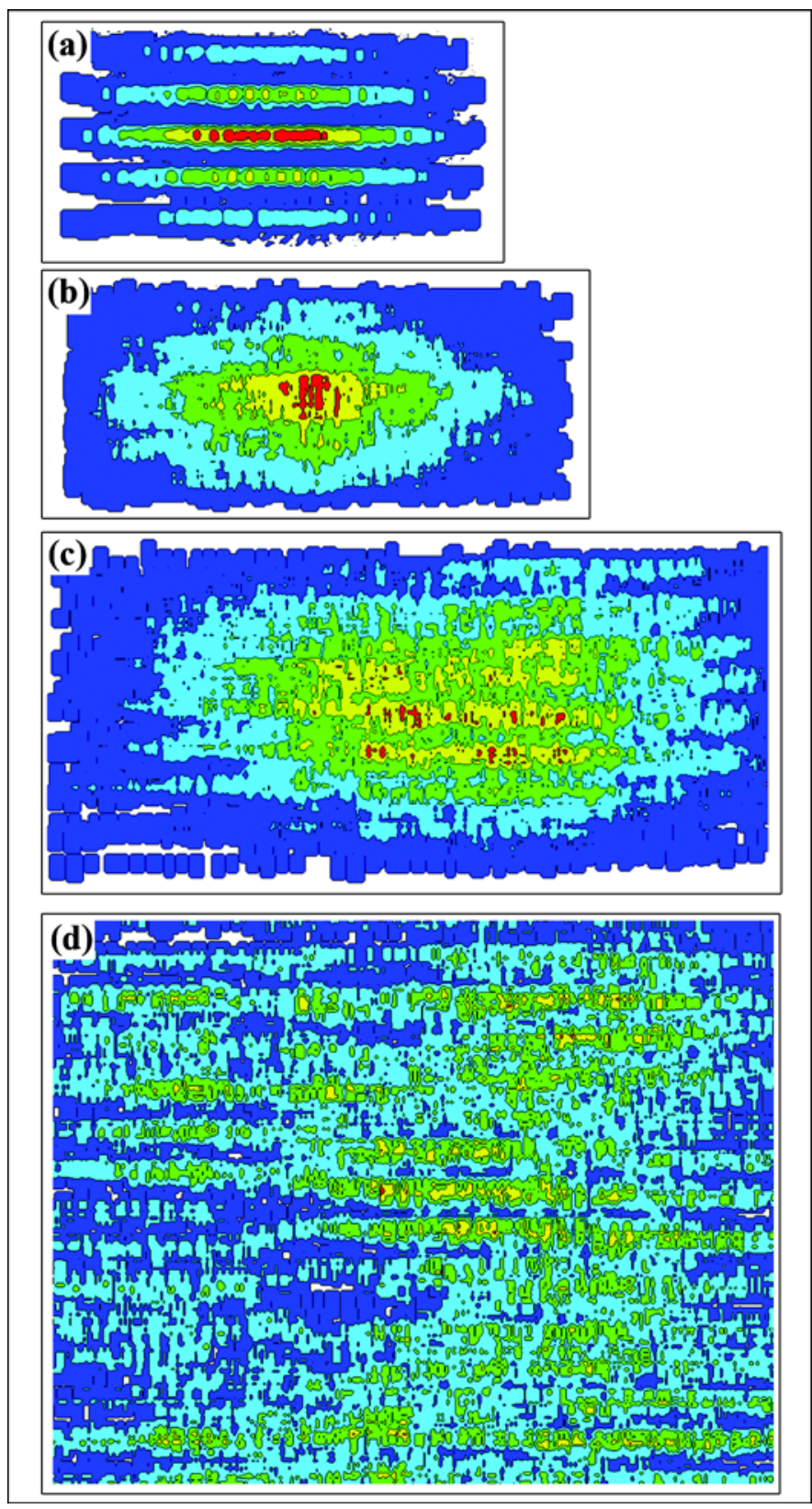

Figure 17.

(a) Five-line text map produced by computer simulations that required preferred retinal locus (PRL) to land on text characters. (b)-(d) Text maps produced by simulation in which PRL was placed randomly within rectangular area around text: (b) $112 \times 48$ pixel area just surrounding text, (c) $224 \times 96$ pixel area, and (d) $320 \times 240$ pixel area (entire scanning laser ophthalmoscope raster). See Figure 10 for color scale.

pixel-area that just enclosed the three-line text with the smallest characters used in experiments. For comparison, the 150-shift text map produced by the first simulation is shown in Figure 17(a). Both the random-scan and character-constrained maps had a region of highest text occupancy in the center of the map but otherwise were quite different. Random-scan and character-constrained maps increasingly differ as the area in which the random shifts occur increases. For example, Figure 17(c) shows the text map from random scans within a $224 \times 96$ pixel area (each dimension twice that of Figure 17(b)) and Figure 17(d) shows the equivalent to the full SLO raster $(320 \times 240$ pixels) in Figure 17(c). Some horizontal line structure is seen, but no well-defined retinal locus of greatest text occupancy or five-line structure exists. Thus, realistic text maps cannot be produced if the tPRL (or fovea) moves randomly within a rectangular area.

The third computer simulation produced text maps that would occur if more than one PRL were used for scanning text. The simulation shifted from one PRL to another during the text scan sequence that was otherwise identical to the first simulation. Figure 18 shows a simulation of three tPRLs. The simulated subject scanned the text with a tPRL that was first to the left, then to the right, and finally, above the scotoma (the shaded area in center). Three distinct fiveline maps with a red region of highest quintile text occupancy are seen in the appropriate locations.

\section{DISCUSSION}

The retinal text maps described here show the relative amount of time a retinal area was exposed to text while subjects were reading. We found that the top quintile of text occupancy surrounds the foveal center in subjects without vision loss. The most parsimonious interpretation

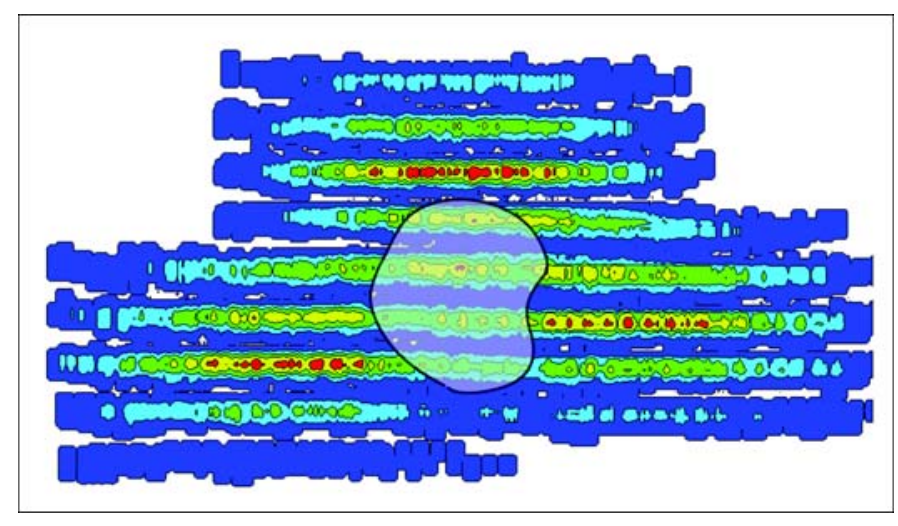

Figure 18.

Simulated text map produced by computer simulation in which three different preferred retinal loci scanned text. Shaded area in center represents scotoma. See Figure 10 for color scale. 
of this result is that these subjects used the fovea to scan text when reading. Similarly, the top quintile of text occupancy in subjects with macular scotomas was in various locations relative to the scotoma and nonfunctional fovea. Again, the most parsimonious interpretation is that the retinal area of highest text occupancy is used for reading text. Thus, retinal text maps show the retinal area(s) that subjects used to process text characters and words during reading.

An important finding is that the PRL used for fixating (fPRL) was not necessarily used for scanning text (tPRL). Five of the eight subjects with scotomas used the same retinal locus to scan text and fixate. The other three subjects with scotomas, however, used separate retinal regions for these two functions. Thus, one cannot identify the retinal locus for fixation of a subject with scotomas and assume that he or she uses the same locus for scanning text. This finding may have important consequences for low-vision rehabilitation.

Retinal text maps do not provide information about the eye movement pattern that produced them. For example, orderly "staircase" eye movements (Figure 3) are not required for producing a typical text map such as in Figure 12. Similar text maps can be produced by simulations in which disorderly sequences of retinal shifts ("saccades") bring the fovea (or tPRL) to text characters. Entirely random retinal shifts that do not direct the gaze to text characters, however, do not result in realistic text maps as shown in Figure 17.

In the present study, we produced text maps based on analysis of a single three-line sentence read by each subject. We believe that significantly different text maps are unlikely to result from reading different sentences, but this must be examined experimentally. Further, combining data from reading multiple sentences or paragraphs to form a single text map may be useful.

Finally, the method for producing retinal text maps described here may help evaluate rehabilitation techniques for training use of a particular retinal area for reading [3-4] or for simply identifying the retinal area an individual uses without training. This is particularly true since the retinal locus that is "reading" extended text at any given moment is difficult or impossible to deduce. Furthermore, our finding that some subjects use different retinal loci for scanning text and fixating means that one cannot assume that the fPRL is also used for scanning text. Consequently, eccentric-viewing training procedures that emphasize fPRL use may not improve reading performance. This finding may explain why reports on efficacy of eccentric-viewing training for reading are inconclusive and often contradictory. The retinal textmapping methodology may also demonstrate whether a subject has "multiple" tPRLs [7-8]. We did not find examples of multiple tPRLs in our study, but the simulations indicate that if a subject were to use multiple tPRLs our text-mapping methodology would demonstrate them.

\section{CONCLUSIONS}

We have developed an SLO image processing and measurement method for determining the retinal area that scans text when someone reads. Not surprisingly, individuals with unimpaired foveal vision use their fovea to scan text and fixate individual objects. Individuals with central scotomas due to macular degeneration, however, do not fixate objects with their nonfunctional foveas, but rather with a retinally eccentric area, the fPRL. We have found that some subjects with scotomas also use their fPRL to scan text. Other subjects, however, scan text with the tPRL, a retinal area that is different from the fPRL. Using the method we described, one can determine whether a given subject with scotomas uses the fPRL or a distinct tPRL to scan text. Identifying the retinal tPRL with our method could aid evaluation of text scanning in subjects with scotomas and evaluation of reading rehabilitation programs, such as those that aim to "train" a new retinal locus for text scanning.

\section{ACKNOWLEDGMENTS}

This material was based on work supported by the Department of Veterans Affairs, Rehabilitation Research and Development Service, grant C2029R.

The authors have declared that no competing interests exist.

\section{REFERENCES}

1. Cummings RW, Whittaker SG, Watson GR, Budd JM. Scanning characters and reading with a central scotoma. Am J Optom Physiol Opt. 1985;62(12):833-43.

[PMID: 4083327]

2. Goodrich GL, Quillman RD. Training eccentric viewing. J Vis Impair Blind. 1977;71(9):377-81. 
3. Nilsson UL, Frennesson C, Nilsson SE. Location and stability of a newly established eccentric retinal locus suitable for reading, achieved through training of patients with a dense central scotoma. Optom Vis Sci. 1998;75(12):873-78. [PMID: 9875992]

4. Nilsson UL, Frennesson C, Nilsson SE. Patients with AMD and a large absolute central scotoma can be trained successfully to use eccentric viewing, as demonstrated in a scanning laser ophthalmoscope. Vision Res. 2003;43(16):1777-87. [PMID: 12818347]

5. Timberlake GT, Peli E, Essock EA, Augliere RA. Reading with a macula scotoma. II. Retinal locus for scanning text. Invest Ophthalmol Vis Sci. 1987;28(8):1268-74. [PMID: 3610545]

6. Timberlake GT, Sharma MK, Grose SA, Gobert DV, Gauch JM, Maino JH. Retinal location of the preferred retinal locus relative to the fovea in scanning laser ophthalmoscope images. Optom Vis Sci. 2005;82(3):177-87.

[PMID: 15767869]

7. Safran AB, Duret F, Issenhuth M, Mermoud C. Full text reading with a central scotoma: Pseudo regressions and pseudo line losses. Br J Ophthalmol. 1999;83(12):1341-47. [PMID: 10574811

8. Déruaz A, Whatham AR, Mermoud C, Safran AB. Reading with multiple preferred retinal loci: Implications for training a more efficient reading strategy. Vision Res. 2002; 42(27): 2947-57. [PMID: 12450504]

Submitted for publication June 17, 2005. Accepted in revised form December 7, 2005. 